\title{
Introduction à la publication des actes du colloque
} Formes vivantes

\section{Introduction to the collected papers of the symposium Living forms}

\author{
Jean-Charles Hameau ${ }^{1}$ \\ ${ }^{1}$ Conservateur du patrimoine, Musée national Adrien Dubouché, Limoges \\ jean-charles.hameau@limogesciteceramique.fr
}

RÉSUMÉ. Ce numéro spécial de la revue Arts et sciences est consacré aux actes du colloque Formes vivantes qui s'est tenu à Limoges les 27 et 28 janvier 2020. Cet événement s'inscrivait dans la programmation culturelle de l'exposition éponyme présentée au musée national Adrien Dubouché du 9 octobre 2019 au 10 février 2020. Cherchant à mettre en lumière la présence du vivant dans la céramique de la Renaissance à nos jours, l'exposition s'organisait autour d'un parcours rythmé par un dialogue entre arts et sciences et a permis d'étudier les liens qui unissent une inspiration organique et une matière minérale. Des décors naturalistes de Bernard Palissy aux céramiques biomédicales imprimées en 3D, l'existence d'une proximité spécifique entre la céramique - en tant que matière et technique - et le monde du vivant a ainsi été mise en exergue par des œuvres patrimoniales, des créations contemporaines et des objets scientifiques.

ABSTRACT. The present publication gathers the collected papers of the symposium Living Forms that took place in Limoges on January 27th and 28th 2020. This event was linked to the eponymous exhibition shown at the musée national Adrien Dubouché between October 9th 2019 and February 10th 2020. It aimed to enlight the presence of the living world in ceramic from the Renaissance up to nowadays, within a dialog crossing arts and sciences. From Bernard Palissy's naturalistic ornament to 3D printed ceramic prosthesis, specific similarities between living organisms and mineral matter were thus explored through museum collections, contemporary artworks and scientific items.

MOTS-CLÉS. céramique, vivant, exposition, colloque, Limoges, musée national Adrien Dubouché.

KEYWORDS. ceramic, living world, exhibition, symposium, Limoges, musée national Adrien Dubouché.

\section{Introduction à la publication des actes du colloque Formes vivantes}

Ce numéro spécial de la revue Arts et sciences est consacré aux actes du colloque Formes vivantes qui s'est tenu à Limoges les 27 et 28 janvier 2020. Cet événement s'inscrivait dans la programmation culturelle de l'exposition éponyme présentée au musée national Adrien Dubouché du 9 octobre 2019 au 10 février 2020. Cherchant à mettre en lumière la présence du vivant dans la céramique de la Renaissance à nos jours, l'exposition s'organisait autour d'un parcours rythmé par un dialogue entre arts et sciences et a permis d'étudier les liens qui unissent une inspiration organique et une matière minérale. Des décors naturalistes de Bernard Palissy aux céramiques biomédicales imprimées en 3D, l'existence d'une proximité spécifique entre la céramique - en tant que matière et technique - et le monde du vivant a ainsi été mise en exergue par des œuvres patrimoniales, des créations contemporaines et des objets scientifiques. 


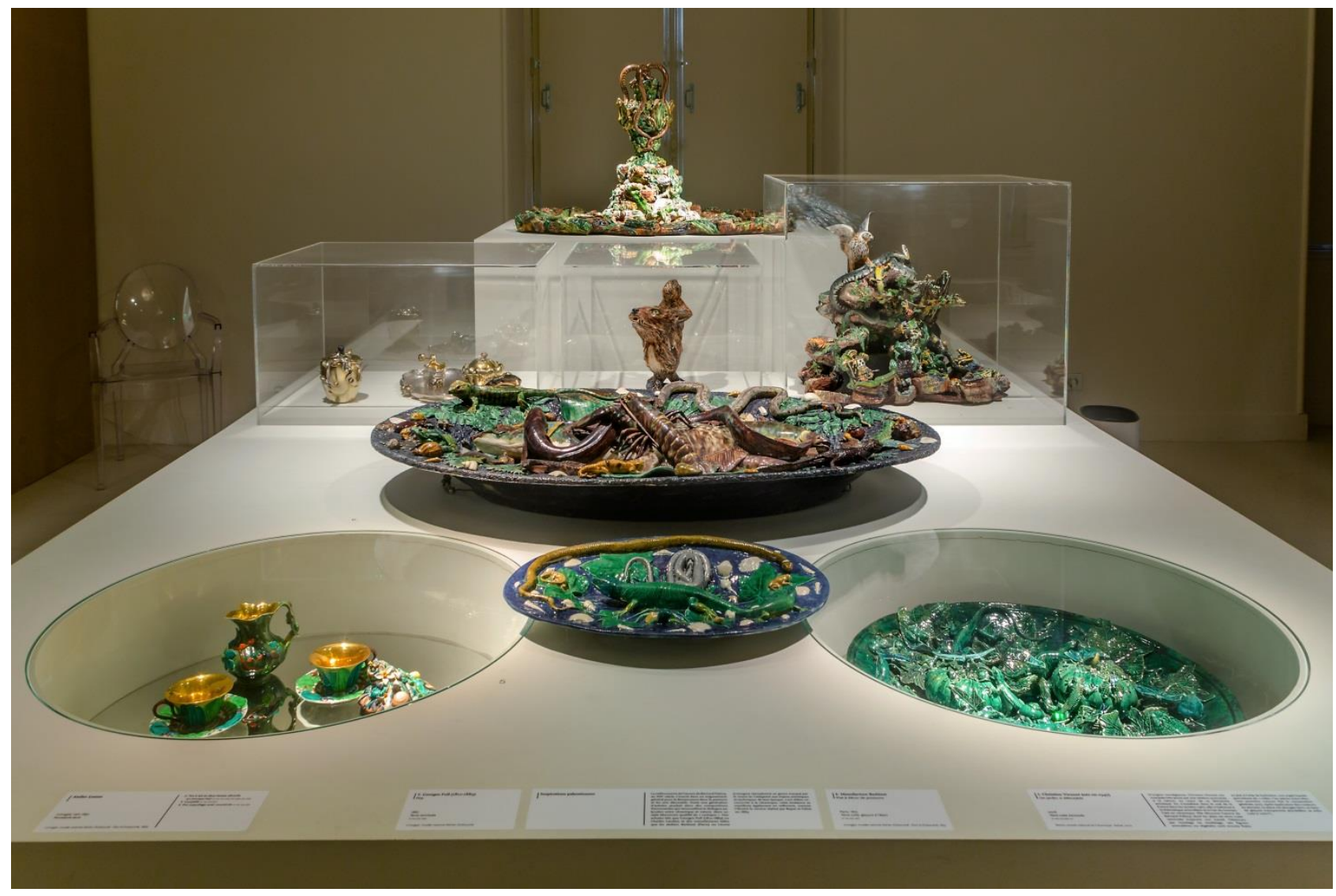

Vue de l'exposition Formes vivantes, première partie intitulée « Naturalismes », photo : Frédérique Avril.

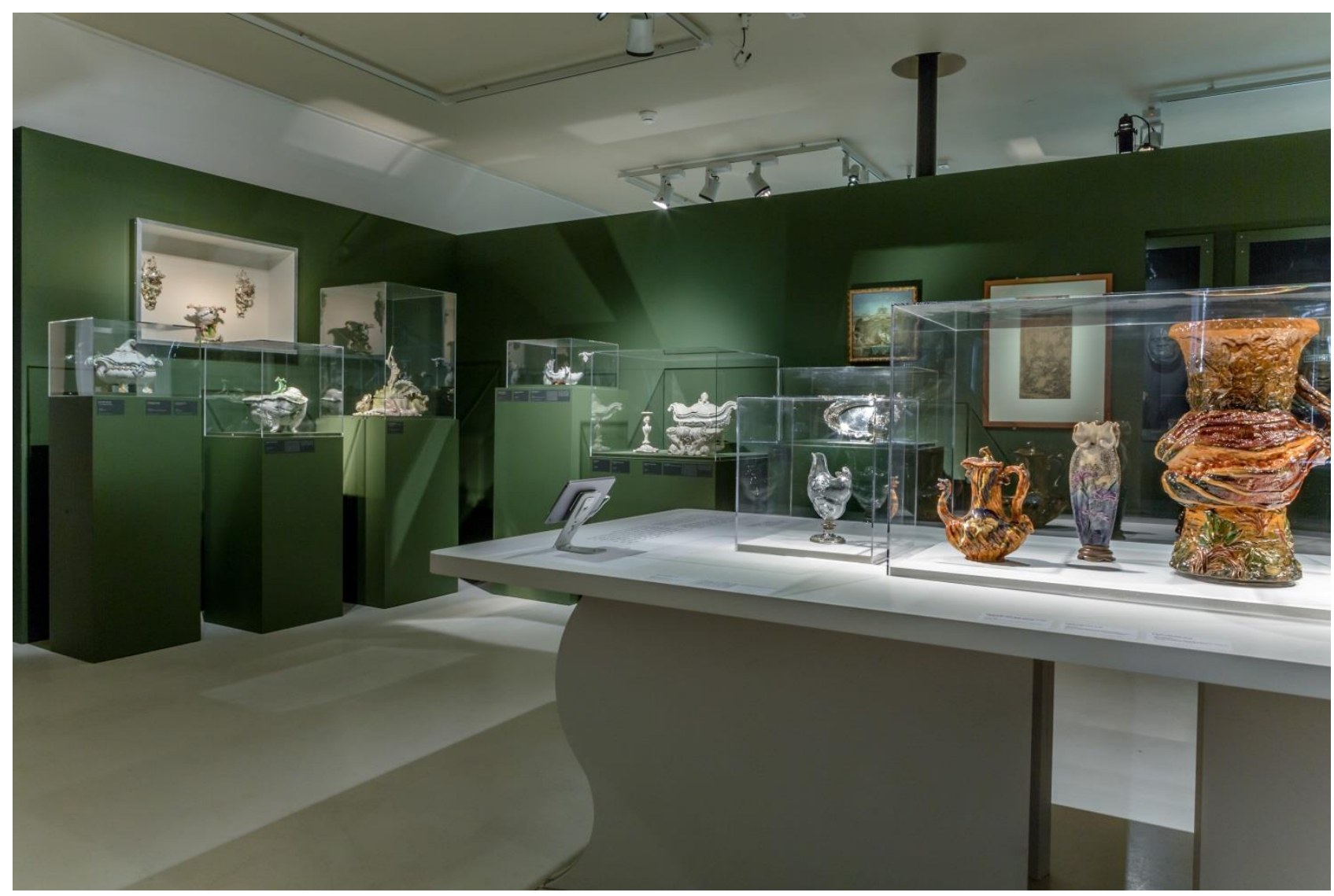

Vue de l'exposition Formes vivantes, deuxième partie intitulée «Imaginaires organiques », photo : Frédérique Avril. 


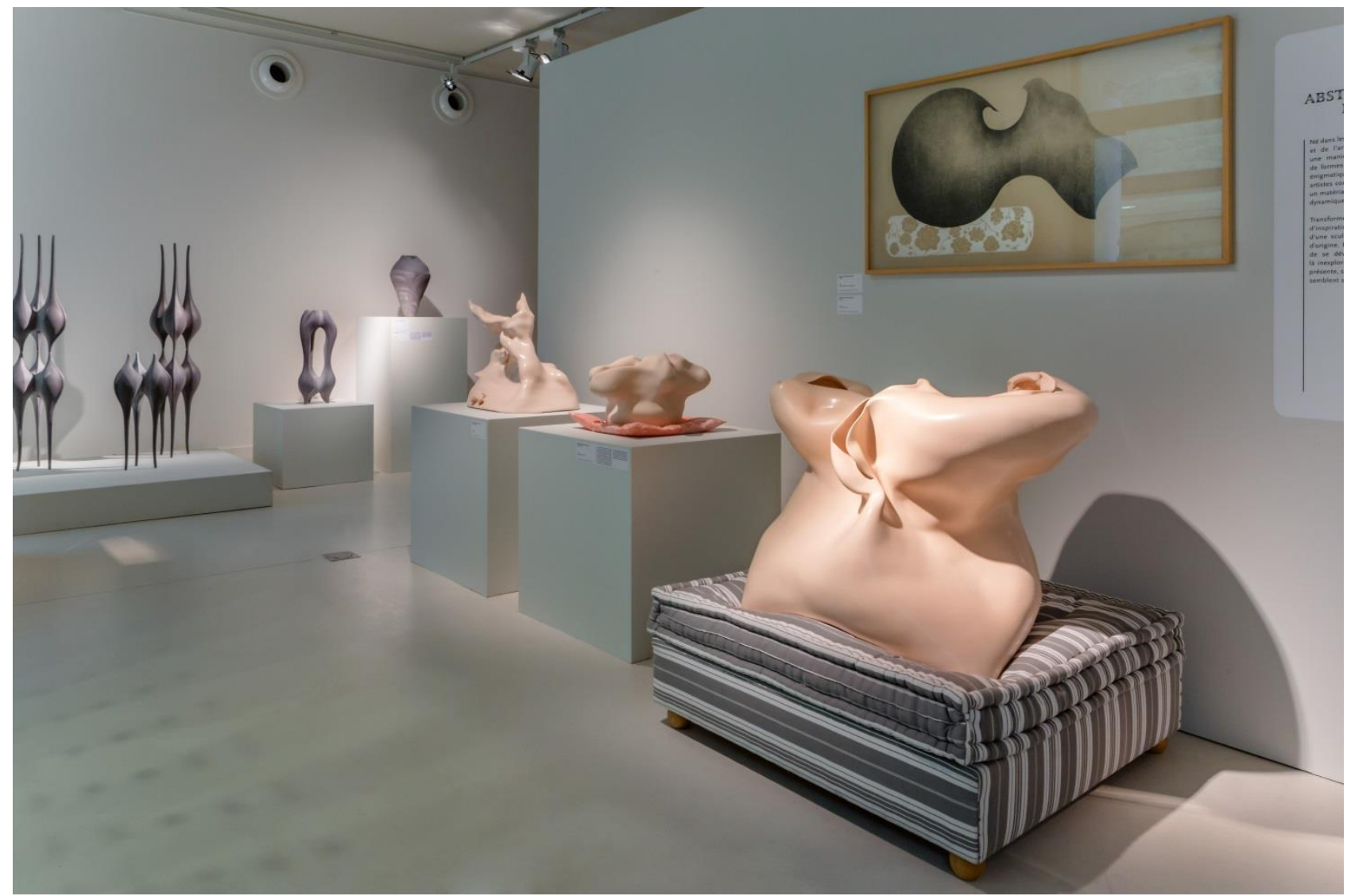

Vue de l'exposition Formes vivantes, deuxième partie intitulée “Imaginaires organiques », photo : Frédérique Avril.

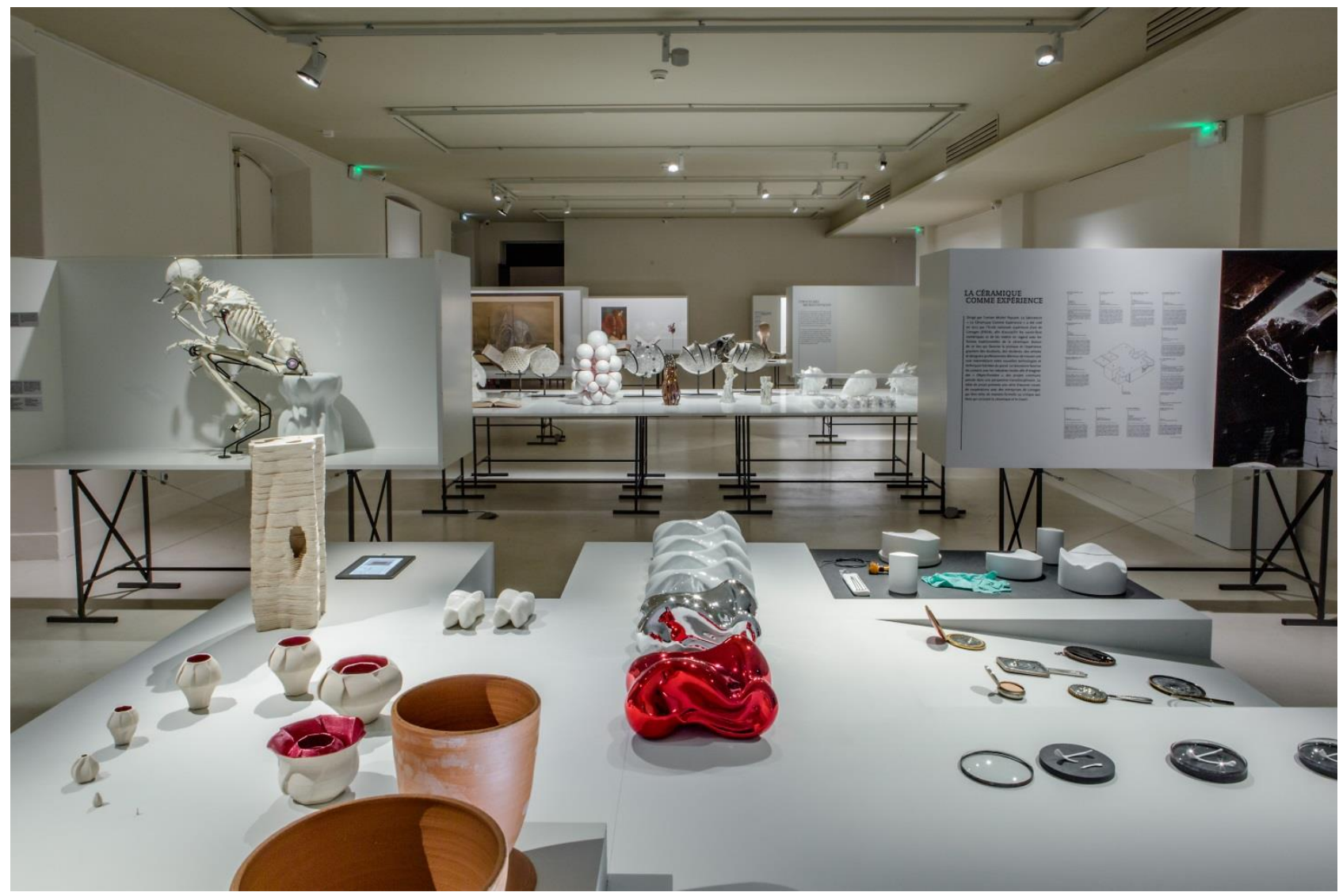

Vue de l'exposition Formes vivantes, troisième partie intitulée "À l'intérieur du vivant », photo : Frédérique Avril. 
L'exposition a donné lieu à la publication d'un premier article dans la revue Arts et sciences en mai $2019^{1}$, puis d'un catalogue ${ }^{2}$ qui rend compte d'une recherche originale menée dans une perspective transdisciplinaire. Composé d'articles et de notices d'œuvres, ce dernier ouvrage illustre la diversité des acteurs concernés par ce rapprochement a priori paradoxal entre le vivant et une matière minérale cuite à des températures incompatibles avec la survie des organismes animaux ou végétaux. Malgré la richesse du catalogue, il nous a semblé indispensable de compléter cette étude par un colloque, au sein duquel ont été invités davantage de chercheurs, d'artistes, d'historiens de l'art et de scientifiques afin d'offrir une place plus importante à leurs travaux.

Nous tenons à remercier chaleureusement tous les intervenants qui ont participé à ce colloque. Leur intérêt, leur expertise et leurs contributions respectives ont permis de faire de ces deux journées un moment intense et stimulant marqué par un esprit de partage des connaissances et de curiosité à l'égard de champs disciplinaires variés. Nous remercions tout aussi vivement les partenaires du musée dans l'organisation du colloque, à savoir l'université de Limoges et la Ville de Limoges, sans le concours desquels l'événement n'aurait pu se dérouler et accueillir du public dans de bonnes conditions. Ce colloque fut le premier organisé par le musée national Adrien Dubouché depuis sa réouverture en 2012. Il concrétise à la fois la volonté de l'établissement d'être à l'initiative de projets de recherche originaux en histoire de l'art et d'étudier la céramique en relation avec des enjeux culturels et sociétaux contemporains.

Nous souhaitons également remercier la revue Arts et Sciences et sa rédactrice en chef MarieChristine Maurel pour son enthousiasme, sa disponibilité, son aide dans l'aboutissement de ce projet. La publication des actes du colloque Formes vivantes dans cette revue est non seulement en parfait accord avec l'ambition de cette dernière de contribuer aux rapprochements entre arts, sciences humaines et sciences dures, mais elle offre également une visibilité en ligne et gratuite au colloque en parfaite complémentarité avec le catalogue.

Au-delà du strict apport de contenus complémentaires, il s'agissait, grâce au colloque, de faire vivre le projet, de l'animer par les échanges, les rencontres et les débats fructueux qu'il a pu susciter. Rétrospectivement, la réunion physique dans un même espace de chercheurs passionnés et d'un public curieux qui a pu exister à cette occasion nous semble d'autant plus précieuse que la crise sanitaire a stoppé net ce type d'événement immédiatement après la fin de l'exposition. Comme de très nombreux projets culturels, la publication de ces actes a malheureusement été retardée en raison d'un fait biologique inédit dans l'histoire de l'humanité. Mais le virus de la Covid-19 n'est pas le seul produit du hasard des mutations, il est l'un des nombreux symptômes d'une crise écologique majeure et rend d'autant plus légitime les initiatives cherchant à repenser le monde du vivant, la place qu'y occupe aujourd'hui l'humain et les rapports qui lient ce dernier à son environnement. Le problème est non seulement politique et scientifique mais aussi esthétique, comme l'écrit le philosophe Baptiste Morizot lorsqu'il évoque la «crise de la sensibilité au vivant ${ }^{3}$ », qui concerne tout un chacun.

Si ce chantier global est à la fois extrêmement urgent et l'affaire de toutes et tous, quelles sont les pistes réflexives qui peuvent être ouvertes par la céramique en général et la porcelaine en particulier, au sein d'un musée spécialisé dans cette technique et sur un territoire historiquement marqué par sa pratique aux échelles artistique, artisanale et industrielle ? Dans quelle mesure l'étude des formes

1 Jean-Charles Hameau et Kimberley Harthoorn, "Formes vivantes », dans Arts et sciences, revue en ligne, mai 2019, https://www.openscience.fr/Formes-vivantes, consulté le 09 juillet 2021.

2 Jean-Charles Hameau et Céline Paul (dir.), Formes vivantes, catalogue de l'exposition, musée national Adrien Dubouché, Silvana Editoriale, Milan, 2019. 
est-elle un terrain commun aux artistes et aux scientifiques ? En quoi la matière argileuse, base de toute céramique, permet-elle de remettre en cause les frontières connues du vivant ? Que peut nous apprendre la céramique sur les origines du monde et de la matière ? Le caractère vertigineux de ces questions semble d'autant plus grand que la céramique est traditionnellement considérée comme un art décoratif par nature éloigné de sujets politiques, de débats épistémologiques ou philosophiques.

La peau émaillée d'une porcelaine ou d'un grès, spectaculairement modifiée par l'action du feu, invite précisément à réviser ce jugement. La céramique peut en effet être rapprochée de la notion de « zone critique » employée notamment par Bruno Latour pour décrire la mince pellicule à la surface de la planète sur laquelle se déploie un réseau dense et complexe d'interactions entre le sol, l'eau et le vivant dont l'analyse requiert le croisement des disciplines, des approches et des outils ${ }^{4}$. L'importance accordée à ce qui se joue sur la couche extérieure de la Terre fait écho à l'attention portée par le céramiste aux effets de surface. Ce constat contredit le rapport moral hérité de Platon et diffusé par le modernisme opposant le décor (dévalorisé, considéré comme une dissimulation de la vérité) au contenu (conceptuel, spirituel ou matériel) valorisé en tant qu'essence d'une chose ${ }^{5}$. Dépasser la hiérarchie des genres pour envisager la céramique comme une «zone critique » permet ainsi de situer cet art dans l'espace ouvert de la «natureculture ${ }^{6}$ » et de l'envisager comme un laboratoire d'étude du monde.

L'étude de la céramique au prisme du vivant conduit également à penser le monde en termes d'écologie et à refaire une place à la matière longtemps dévalorisée par une culture occidentale accordant le primat à l'idée. Au-delà du parallélisme entre la plasticité de l'argile et la capacité du vivant à mémoriser et à se transformer en même temps ${ }^{7}$, la céramique permet d'envisager les liens réciproques qui unissent le biologique et le minéral. Le philosophe François Dagognet soulignait, à ce titre, le miracle accompli par la pierre lithographique « capable d'une prouesse, celle de recevoir, puis de conserver ce que nous avons inscrit sur elle, enfin de nous le restituer intégralement. (Elle l'améliore même, l'orchestre). La pierre lithographique - la vraie pierre philosophale ! - réussit ce que, mutatis, mutandis, notre cerveau archiviste atteint dans son paroxysme (la mémoire matérialisée). [...] Ne faut-il pas revoir nos définitions ou nos catégories, puisque la matière enregistre les moindres souffles et nous les restitue $?^{8} \gg$ Comment ne pas rapprocher la sensibilité des pierres qu'il évoque des techniques employées par les céramistes pour faire advenir des formes, des couleurs et des textures qui sont autant de fruits d'un dialogue avec l'organique ?

Afin d'explorer le plus largement possible les pistes ouvertes par le sujet du colloque, la parole a été donnée à des invités issus, comme on l'a dit, d'univers très variés. La grande hétérogénéité - en termes de longueur, de forme, de ton et de contenu - des articles de ce numéro s'explique ainsi et restitue la diversité des approches, des plus sensibles aux plus théoriques, adoptées par les intervenants qui ont animé ces journées. Les contributions sont ici regroupées en trois parties qui correspondent aux trois thématiques abordées lors du colloque.

\footnotetext{
4 Bruno Latour, Où atterir ?, Éditions la découverte, Paris, 2017, p, 101. Voir également la plateforme de recherche et de création « Zone critique » créée par Frédérique Aït-Touati : https://zonecritique.org/inside/, consultée le 14 janvier 2021.
}

5 Voir Thomas Golsenne, "L'ornement aujourd'hui », dans Images Re-vues :

http://journals.openedition.org/imagesrevues/2416, consulté le 14 janvier 2021.

6 Donna Haraway, cité par Delphine Gardey, « Donna Haraway : poétique et politique du vivant », Cahiers du Genre, N55, 2013, L'Harmattan, Paris, p. 171-194.

7 Voir Formes vivantes, Op. cit. p. 21-22.

8 François Dagognet, Des détritus, des déchets, de l'abject, une philosophie écologique, Institut Synthélabo, Le Plessis-Robinson, 1997, p. 46-48. 
Au sein d'une première partie intitulée « Matière céramique et matière organique », la frontière entre matière vivante et matière inerte est remise en question, autant par des hypothèses scientifiques que par des intuitions artistiques ; il s'agit d'envisager la céramique dans sa matérialité, comme un terrain d'étude original des liens qui unissent le vivant et les corps dits «inertes ». La seconde partie est dédiée aux «Perspectives techniques et culturelles» ouvertes par la problématique du colloque. Les prothèses en céramique biomédicale ou les œuvres biomorphiques imprimées en 3D invitent à penser le vivant sous l'angle du dépassement de la division entre nature et culture. Comment la céramique et les œuvres bio-inspirées peuvent-elles contribuer à un débat d'actualité sur la prise en compte du vivant par les êtres humains ? Enfin, la dernière partie intitulée «Vie des formes », en hommage à l'historien d'art Henri Focillon, abordera la notion de forme vivante en rapprochant les outils méthodologiques de l'histoire de l'art et ceux de l'étude scientifique de la morphogenèse.

Depuis la fermeture de l'exposition en février 2020, d'autres projets touchant le vivant, son histoire ou son actualité dans les arts et les sciences ont vu le jour. On songe par exemple à l'exposition Les origines du monde au Musée d'Orsay ${ }^{9}$, à l'exposition La vie à elle-même au Centre international d'art du paysage de Vassivière ${ }^{10}$, aux rencontres du Parlement des liens ${ }^{11}$ organisées au Centre Pompidou ou encore à l'ouverture à l'École nationale supérieure des beaux-arts de Paris d'une chaire intitulée « Habiter le paysage - l'art à la rencontre du vivant ${ }^{12}$ ».

Le rapprochement fertile entre vivant et céramique continue d'être exploré par de nombreux événements : citons entre autres l'exposition personnelle de Claire Lindner à Vallauris, Lanscape within $^{13}$, ou l'exposition au musée des beaux-arts de Lyon Par le feu la couleur, au sein de laquelle une partie est consacrée à la manière dont l'anthropocène irrigue la création contemporaine ${ }^{14}$. Ces quelques exemples témoignent de l'actualité des recherches menées dans le cadre de l'exposition Formes vivantes, dont nous sommes fiers d'annoncer qu'elle sera reprise au musée national de céramique de Sèvres en 2022. Gageons que les actes présentement publiés offriront aux chercheurs des ressources utiles et à toutes les personnes intéressées par le sujet des clés pour en approfondir la lecture.

9 Musée d’Orsay, du 19 mai au 18 juillet 2021.

10 CIAP Vassivière, du 13 juin au 5 septembre 2021.

11 Voir les ressources en ligne : https://www.leparlementdesliens.fr, consulté le 09 juillet 2021.

12 Voir la présentation de la chaire : https://www.beauxartsparis.fr/fr/chaire/chaire-habiter-le-paysage-lart-la-rencontre-duvivant, consulté le 09 juillet 2021.

13 Exposition personnelle de Claire Lindner, Landscape within, EACV, 2 juillet-2 octobre 2021.

14 Voir Jean-Charles Hameau, "Formes mutantes », dans Salima Hella (dir.), Par le feu la couleur, céramiques contemporaines, catalogue de l'exposition, musée des beaux-arts de Lyon, Snoeck, 2021. 